\title{
Factores asociados a la histología de masa residual retroperitoneal post quimioterapia en tumor testicular de células germinales
}

\section{Factors Associated with the Histology of Retroperitoneal Residual Mass Post Chemotherapy in Testicular Germ Cell}

\section{Tumor}

Lynda Torres $^{10}$ Jose Gustavo Ramos ${ }^{2}$ Rodolfo Varela ${ }^{2}$ Fabian Godoy ${ }^{2}$ Paola Andrea Orrego ${ }^{3}$ AysaVanessa Mosquera $^{3}$ Sandra Viviana Pulido Vecino ${ }^{3}$

\footnotetext{
1 Médica Uróloga. Pontificia Universidad Javeriana, Bogotá, Colombia

2 Médico Urólogo, Urología Oncológica, Instituto Nacional de Cancerología, Bogotá, Colombia

3 Médica residente, Universidad Militar Nueva Granada, Bogotá, Colombia
}

Urol Colomb 2020;29:235-239.

Resumen

Palabras Clave
- neoplasias
testiculares
- disección de ganglios
linfáticos
retroperitoneales
- necrosis
- teratoma

Abstract
Objetivo El objetivo de este estudio es describir las características y factores relacionados con la histología de la masa residual postquimioterapia en pacientes con tumor de células germinales de origen testicular sometidos a linfadenectomía retroperitoneal durante 12 años de seguimiento.

Métodos Retrospectivamente se recolectaron datos clínicos, quirúrgicos y patológicos de la historia clínica de los pacientes en un centro de referencia de manejo de cáncer durante un periodo de 12 años. Se estimó la asociación entre los datos recolectados con la histología del tumor residual post quimioterapia.

Resultados Se incluyeron 64 pacientes, la edad promedio fue 28.1 años, el tamaño promedio de masa residual fue de $6.7 \mathrm{~cm}$. La histología de la masa residual fue teratoma en $60.9 \%$, necrosis $26.5 \%$ y tumor viable $12.5 \%$. El grupo pronóstico tiene asociación con la histología de la masa retroperitoneal. Las masas con histología de necrosis tuvieron menor tamaño con media $6.5 \mathrm{~cm}$ mientras que otras histologías tuvieron tamaño promedio de la masa residual de $10.4 \mathrm{~cm}$.

Conclusiones La LNDRP-PC es el estándar de tratamiento en masas residuales retroperitoneales después de quimioterapia y puede generar sobre-tratamiento hasta en $50 \%$ de los casos. El teratoma en la histología testicular está relacionado mayor tamaño de la masa residual retroperitoneal. Las características histológicas de la masa residual son comparables con otras series.

Objective The aim of the study is to describe factors and characteristics related with the post-chemotherapy residual mass histology in patients who undergoing RPLND. received

May 31, 2020

accepted

September 14, 2020
DOI https://doi.org/

10.1055/s-0040-1721357.

ISSN 0120-789X.

e ISSN 2027-0119.
Copyright (c) 2020, Thieme Revinter Publicações Ltda.., Rua do Matoso 170 , Rio de Janeiro, RJ, CEP 20270-135, Brazil. Todos los derechos reservados.

\section{License terms}

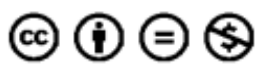




\section{Keywords}

- testicular neoplasms

- retroperitoneal lymph node dissection

- necrosis

- teratoma
Methods Clinical, surgical and pathological data were retrospectively recorded from medical records of patients with diagnosis of germ cell tumor and post-chemotherapy residual retroperitoneal tumor in a cancer referral center over a period of 12 years. The association between the data collected and the post-chemotherapy residual tumor histology was assessed.

Results Sixty-four patients were included with average age of 28.1 years. The mean residual mass size was $6.7 \mathrm{~cm}$. The histology of the mass was teratoma in $60.9 \%$, necrosis $26.5 \%$ and viable tumor $12.5 \%$. Prognostic group was associated with the mass histology. Mass with necrosis histology was smaller with mean size of $6.5 \mathrm{~cm}$, while other histologies had an average mass size of $10.4 \mathrm{~cm}$.

Conclusion PC-RPLND is the standard of management of retroperitoneal residual mass after chemotherapy and could over treat in up to $50 \%$ of cases. Teratoma component in testicular histology is related to increase size of the residual mass. Histology findings of the retroperitoneal mass are comparable with other series.

\section{Introducción}

El tumor testicular es la malignidad más común en hombres entre los 15-35 años y su incidencia se ha duplicado en los últimos 40 años. Al momento del diagnóstico 10-30\% se presentan con enfermedad metastásica. ${ }^{1,2}$

La mayoría de los pacientes pueden quedar curados de la enfermedad pero hay controversías en el manejo de las masas residuales después de quimioterapia. En el caso de paciente con tumores metastásicos germinales no seminomatosos que recibieron quimioterapia el manejo de la masa residual es con LNDRP-PC (cuando los marcadores son negativos y la masa residual mayor de $1 \mathrm{~cm}$ en la mayor dimensión), en el caso de tumores metastásicos germinales seminomatosos se realiza la evaluación con tomografía con emisión de positrones cuando la masa residual es mayor de $3 \mathrm{~cm}$ y cuando se documenta que es metabolicamente activa se realiza manejo quirúrgico. ${ }^{3,4}$

Cuando se realiza la resección completa de la masa residual después de quimioterapia los resultados a largo plazo en cuanto a supervivencia libre de enfermedad pueden tener una tasa hasta de $95 \%$, siendo de impacto en quienes tienen en la histología tumor viable o teratoma; ${ }^{5}$ sin embargo hasta en el 50\% de los casos la histología puede corresponder a necrosis/fibrosis y en este caso los pacientes son sometidos a cirugía innecesariamente y a las posibles complicaciones asociadas a esta teniendo en cuenta la complejidad de la intervención. ${ }^{6}$ Predecir la histología del tumor residual con exactitud podría evitar la cirugía en quienes tienen necrosis/fibrosis generando un reto al seleccionar los pacientes que se beneficiaran de LNDRP-PC. El objetivo de este estudio es describir las características y factores relacionados con la histología de la masa residual postquimioterapia en pacientes con tumor de células germinales de origen testicular sometidos a linfadenectomía retroperitoneal durante 12 años de seguimiento.

\section{Métodos}

Se realizó un estudio retrospectivo en el que se incluyeron hombres de cualquier edad con diagnóstico histológico de tumor testicular de células germinales que fueron sometidos a quimioterapia de primera o segunda línea y llevados a linfadenectomía retroperitoneal citorreductora por masa residual. Se obtuvieron datos entre enero de 2006 y octubre de 2018.

Se evaluaron datos demográficos, marcadores tumorales previos y posteriores a la quimioterapia, histología del tumor germinal testicular, la clasificación TNM, el estadio tumoral y el grupo pronóstico IGCCCG (grupo colaborativo internacional para el cáncer de células germinales), ${ }^{7}$ el número de ciclos de quimioterapia y la necesidad de quimioterapia de segunda línea, el tamaño de la masa previo y posterior a la quimioterapia, la histología de la masa residual retroperitoneal y características quirúrgicas y postquirúrgicas.

El análisis estadístico se realizó con el software Stata 15 (StatCorp, Texas, versión 15.0). Se realizó un análisis de estadística descriptiva, se utilizó la prueba de ANOVA y Mann-Whitney para la comparación de medias entre grupos. La comparación de variables categóricas se realizó mediante la prueba de Chi cuadrado y la correlación entre las variables continuas mediante la correlación Spearman. A los resultados se les aplico la corrección de Bonferroni.

Este estudio se llevó a cabo con la aprobación del comité de ética del Instituto Nacional de Cancerología.

\section{Resultados}

Se incluyeron 64 pacientes, las características de los pacientes se mencionan en la tabla 1. La mayoría (75\%) de los pacientes tenían histología mixta NSGCT (tumor de células germinales no seminomatoso) en la patología inicial, el $60.2 \%$ de los pacientes eran estadio II y el $51.6 \%$ eran S1. Para el grupo NSGCT el $77.9 \%$ eran de buen pronóstico y el 100\% para los SGCT (tumor de células germinales seminomatoso).

Se documentó asociación entre la histología de la masa residual postquimioterapia y el grupo pronóstico: de los que tenían histología de la masa residual necrosis/fibrosis $66.7 \%$ era de buen pronóstico de acuerdo a la clasificación IGCCCG y 
Tabla 1 Características de los pacientes

\begin{tabular}{|c|c|}
\hline Edad años $(n=64)$ & $26.2(16-47) \mathrm{DE}=6.5$ \\
\hline \multicolumn{2}{|l|}{ Estadio clínico (n=64), n (\%) } \\
\hline EC I con recaída & $4(6.2)$ \\
\hline EC II & $39(60.9)$ \\
\hline EC III & $21(32.8)$ \\
\hline \multicolumn{2}{|c|}{ Grupo pronóstico IGCCCG NSGCT* $(n=59)$, \% (92.9) } \\
\hline Bueno & $46(77.9)$ \\
\hline Intermedio & 7 (11.9) \\
\hline Pobre & $6(10.1)$ \\
\hline \multicolumn{2}{|c|}{ Grupo pronóstico IGCCCG SGCT ${ }^{* *}(n=5), n$ \% (7.8) } \\
\hline Bueno & $5(100)$ \\
\hline Intermedio & $0(0)$ \\
\hline \multicolumn{2}{|c|}{ Histología de la orquiectomía $(n=60)$ n $(\%)$} \\
\hline NSGCT mixto & $45(75)$ \\
\hline Seminoma puro & $5(8.3)$ \\
\hline Teratoma puro & $5(8.3)$ \\
\hline Carcinoma embrionario puro & $5(8.3)$ \\
\hline \multicolumn{2}{|c|}{ Estadio S prequimioterapia $(n=64) n(\%)$} \\
\hline SO & $2(3.1)$ \\
\hline S1 & $33(51.6)$ \\
\hline S2 & $14(21.9)$ \\
\hline S3 & $5(7.8)$ \\
\hline Sx & $10(15.6)$ \\
\hline \multicolumn{2}{|l|}{ Quimioterapia n (\%) Rango } \\
\hline $\begin{array}{l}\text { Número de ciclos de primera } \\
\text { línea }(n=64)\end{array}$ & $3.9(1-7) \mathrm{DS}=0.9$ \\
\hline $\begin{array}{l}\text { Número de ciclos de segunda } \\
\text { línea }(n=10)(15.6 \%)\end{array}$ & $3.6(3-4) \mathrm{DS}=0.5$ \\
\hline \multicolumn{2}{|l|}{ Tamaño de la masa $(\mathrm{cm})$} \\
\hline Pre quimioterapia $(\mathrm{CT}) \mathrm{n}=57$ & $5.2(0-18) \mathrm{DE}=4.2$ \\
\hline Post quimioterapia $(\mathrm{CT}) \mathrm{n}=56$ & $6.7(1-28) \mathrm{DE}=4.5$ \\
\hline \multicolumn{2}{|c|}{ Histología de la masa retroperitoneal $n=64 n$ (\%) } \\
\hline Necrosis & $17(26.5)$ \\
\hline Teratoma & $39(60.9)$ \\
\hline Tumor viable & $8(12.5)$ \\
\hline
\end{tabular}

EC, Estadio Clínico, IGCCCG, grupo colaborativo internacional para el cáncer de células germinales, NSGCT, Tumor de Células Germinales no Seminomatoso, SGCT, Tumor de Células Germinales Seminomatoso

de los que tenían en la histología teratoma $84.2 \%$ eran de buen pronóstico. De los paciente que tenían tumor viable en la histología $60 \%$ estaban clasificados como riesgo intermedio $\left(X^{2}=16.37, p=0.01\right)$. No se estableció asociación alguna entre la histología de la masa residual y el grupo de pobre pronóstico. Respecto a los ciclos de quimioterapia y la relación con la histología final tener teratoma en la histología final se asoció a

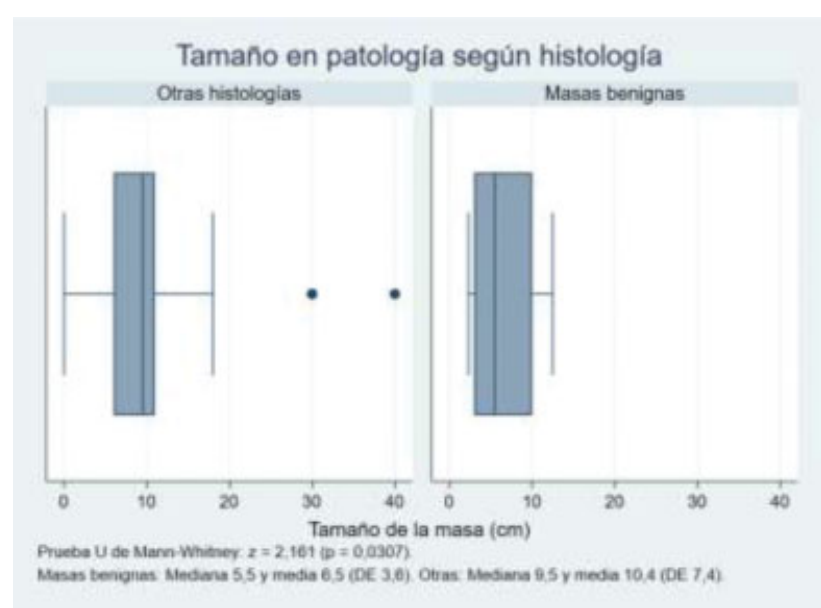

Figura 1 Tamaño de la patología de acuerdo a la histología de la masa residual después de quimioterapia

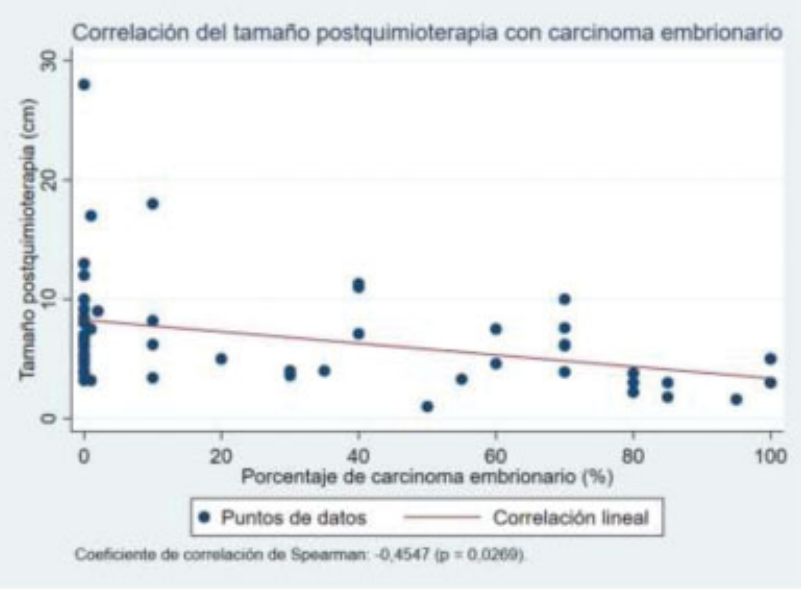

Figura 2 Correlación entre el porcentaje de carcinoma embrionario en la histología testicular y el tamaño de la masa residual después de quimioterapia

recibir menos ciclos de quimioterapia $\mathrm{OR}=0.43 \mathrm{IC} 95 \%(0.22-$ 0.84) $\mathrm{p}=0.014$ Pseudo-R2 8\%.

Las masas residuales benignas tienen menor tamaño en el estudio histológico final comparado con las otras histologías con una diferencia de $3.9 \mathrm{~cm} \mathrm{(} \mathrm{z}=2.161$ $\mathrm{p}=0.0307$ ) (-Figura 1). Adicionalmente quienes tienen teratoma en la histología final tienen mayor tiempo quirúrgico en la LDNRP-PC (mediana de 371 minutos y 307 minutos respectivamente $\mathrm{p}=0.0449$ ).

Existe una correlación inversa entre el tamaño de la masa retroperitoneal y el porcentaje de carcinoma embrionario en la histología testicular (Correlación -0.4 p=0.027) (-Figura 2).

En cuanto al cambio del tamaño de la masa retroperitoneal después la quimioterapia de acuerdo a la histología testicular el componente de teratoma tuvo aumento promedio de $2.28 \mathrm{~cm}$, mientras que quienes tenían seminoma puro en la histología testicular tuvieron reducción promedio de $3.1 \mathrm{~cm}$ del tamaño de la masa residual (tabla 2). 
Tabla 2 Cambio de tamaño de masa retroperitoneal postquimioterapia $(\mathrm{cm})$

\begin{tabular}{|l|l|l|}
\hline Histología testicular & Promedio (DE) & $\mathrm{P}^{*}$ \\
\hline Componente de Teratoma & $2.28(6.08)$ & 0.0429 \\
\hline Teratoma puro & $6.44(11.12)$ & 0.2006 \\
\hline Componente de Seminoma & $1.36(1.36)$ & 0.9113 \\
\hline Seminoma puro & $-3.1(3.6)$ & 0.0175 \\
\hline
\end{tabular}

Tabla 3 Histología de la masa residual retroperitoneal después de quimioterapia

\begin{tabular}{|c|c|c|c|}
\hline Literatura & $\begin{array}{l}\text { Necrosis/ } \\
\text { fibrosis (\%) }\end{array}$ & $\begin{array}{l}\text { Teratoma } \\
(\%)\end{array}$ & $\begin{array}{l}\text { Tumor } \\
\text { viable (\%) }\end{array}$ \\
\hline Leao et al. ${ }^{14}$ & 24 & 66.8 & 9.2 \\
\hline Heidenreich et al. ${ }^{13}$ & 65.9 & 12.4 & 21.6 \\
\hline Aprikian et al. ${ }^{14}$ & 45 & 42.5 & 12.5 \\
\hline Djaladat et al. ${ }^{15}$ & 28 & 39 & 32 \\
\hline Oldenburg et al. $^{16}$ & 67 & 26 & 7 \\
\hline Albers et al. ${ }^{11}$ & 35 & 34 & 31 \\
\hline Ozen et al. ${ }^{17}$ & 33.3 & 45.3 & 21.3 \\
\hline Torres et al. & 27 & 60.3 & 12.7 \\
\hline
\end{tabular}

Comparación con otras cohortes

\section{Discusión}

El manejo actual de lesiones residuales retroperitoneales después de quimioterapia continua siendo controversial. Existe la indicación cuando hay masas residuales $>1 \mathrm{~cm}$ con marcadores negativos en tumor germinal no seminomatoso y cuando en la tomografía con emisión de positrones hay lesiones metabólicamente activas en masas residuales mayores de $3 \mathrm{~cm}$ en el escenario de tumor germinal seminomatoso. ${ }^{3}$ Sin embargo, el $50 \%$ de estos pacientes pueden ser sometidos a un procedimiento innecesario asociado a complicaciones y cirugías concomitantes. ${ }^{8-10}$

La histología de la masa residual postquimioterapia es comparable al de otras series (tabla 3 ), nuestra cohorte hasta el $27 \%$ de los pacientes pueden tener necrosis/fibrosis generando la pregunta de cómo predecir la histología de la masa residual y evitar la LDNRP y sugiriendo que la cohorte analizada está compuesta por pacientes en estadio avanzado de la enfermedad. En otras series se han documentado factores predictores de necrosis como el valor de los marcadores antes de la quimioterapia, el tamaño inicial de la masa retroperitoneal y el porcentaje de reducción de la masa después de la quimioterapia. ${ }^{11,12}$

Varios modelos clínicos se han creado para predecir necrosis/fibrosis en el escenario postquimioterapia pero sin encontrar resultados consistentes que hayan generado cambios en la práctica ya establecida. Vergouwe y colaboradores en 2001 describieron un nomograma en donde toman en cuenta 6 variables: presencia de teratoma en el tumor primario, clasificación del teratoma: diferenciado o indeterminado, niveles de marcadores tumorales prequimioterpia, adicionalmente el nomograma creado por Leao y colaboradores en 2018 incluye presencia de teratoma en la histología inicial, alfa feto proteína pre quimioterapia, tamaño de la masa pre quimioterapia y cambio del tamaño de la masa residual. ${ }^{18,19}$ Dentro de nuestros resultados el componente de teratoma en la histología testicular estuvo relacionado con aumento de tamaño de la masa residual, y este a su vez se relacionó con teratoma en la histología final.

En nuestra cohorte se identificaron otros desenlaces como la asociación entre el número de ciclos de quimioterapia y la histología, documentando que aquellos que tienen teratoma en la histología de la masa residual recibieron 3 o menos ciclos de quimioterapia. Este hallazgo no está documentado en otros estudios y puede ser explicado porque algunos pacientes reciben el manejo con quimioterapia en centros que no son de referencia en manejo de cáncer y varía el número de ciclos administrados. También se documentó que aquellos que tienen componente de teratoma en la patología inicial tienen aumento de la masa residual después de quimioterapia contrario a lo que sucede con aquellos que tienen seminoma puro en la histología inicial quienes tienden a la reducción de la masa retroperitoneal después de la quimioterapia. Otras series han documentado que la reducción del $90 \%$ del tamaño de la masa podría predecir de manera confiable necrosis en la histología e la masa residual. $^{20}$

Este estudio tiene limitaciones al ser retrospectivo, adicionalmente por el tamaño de la muestra y los datos perdidos solo se pudieron establecer asociaciones y no relaciones de causalidad, por lo anterior nuestros hallazgos no se pueden establecer como factores de riesgo o para crear modelos de predicción de la histología de la masa residual después de quimioterapia. Hasta el momento es la cohorte con mayor número de pacientes descrita en Colombia en este escenario clínico.

\section{Conclusión}

La LNDRP-PC es el estándar de tratamiento en masas residuales retroperitoneales y puede generar sobretratamiento en un porcentaje no despreciable de casos. El componente de teratoma en la histología testicular está relacionado con aumento de tamaño de la masa residual. Las características histológicas de la masa residual después de quimioterapia son comparables con los de otras series.

\section{Conflicto de intereses}

Los autores declaran no tener ningún conflicto de intereses.

\section{Referencias}

1 Le Cornet C, Lortet-Tieulent J, Forman D, et al. Testicular cancer incidence to rise by $25 \%$ by 2025 in Europe? Model-based predictions in 40 countries using population-based registry data. Eur J Cancer 2014;50(04):831-839 
2 Bray F, Ferlay J, Soerjomataram I, Siegel RL, Torre LA, Jemal A. Global cancer statistics 2018: GLOBOCAN estimates of incidence and mortality worldwide for 36 cancers in 185 countries. CA Cancer J Clin 2018;68(06):394-424

3 Albers P, Albrecht W, Algaba F, et al; European Association of Urology. Guidelines on Testicular Cancer: 2015 Update. Eur Urol 2015;68(06):1054-1068

4 Motzer RJ, Jonasch E, Agarwal N, et al. Testicular Cancer, Version 2.2015. J Natl Compr Canc Netw 2015;13(06):772-799

5 Heidenreich A, Pfister D, Witthuhn R, Thüer D, Albers P. Postchemotherapy retroperitoneal lymph node dissection in advanced testicular cancer: radical or modified template resection. Eur Urol 2009;55(01):217-224

6 Steyerberg EW, Keizer HJ, Fosså SD, et al. Prediction of residual retroperitoneal mass histology after chemotherapy for metastatic nonseminomatous germ cell tumor: multivariate analysis of individual patient data from six study groups. J Clin Oncol 1995; 13(05):1177-1187

7 International Germ Cell Cancer Collaborative Group. International Germ Cell Consensus Classification: a prognostic factor-based staging system for metastatic germ cell cancers. J Clin Oncol 1997;15(02):594-603

8 Winter C, Pfister D, Busch J, et al. Residual tumor size and IGCCCG risk classification predict additional vascular procedures in patients with germ cell tumors and residual tumor resection: a multicenter analysis of the German Testicular Cancer Study Group. Eur Urol 2012;61(02):403-409

9 Russell CM, Sharma P, Agarwal G, et al. Is percent seminoma associated with intraoperative morbidity during post-chemotherapy RPLND? Can J Urol 2016;23(01): 8127-8134

10 Cary C, Masterson TA, Bihrle R, Foster RS. Contemporary trends in postchemotherapy retroperitoneal lymph node dissection: Additional procedures and perioperative complications. Urol Oncol 2015;33(09):389.e15-389.e21

11 Albers P, Weissbach L, Krege S, et al; German Testicular Cancer Study Group. Prediction of necrosis after chemotherapy of advanced germ cell tumors: results of a prospective multicenter trial of the German Testicular Cancer Study Group. J Urol 2004;171(05):1835-1838

12 Steyerberg EW, Keizer HJ, Sleijfer DT, et al. Retroperitoneal metastases in testicular cancer: role of CT measurements of residual masses in decision making for resection after chemotherapy. Radiology 2000;215(02):437-444

13 Heidenreich A, Thüer D, Polyakov S. Postchemotherapy retroperitoneal lymph node dissection in advanced germ cell tumours of the testis. Eur Urol 2008;53(02):260-272

14 Aprikian AG, Herr HW, Bajorin DF, Bosl GJ. Resection of postchemotherapy residual masses and limited retroperitoneal lymphadenectomy in patients with metastatic testicular nonseminomatous germ cell tumors. Cancer 1994;74(04): 1329-1334

15 Djaladat H, Nichols C, Daneshmand S. Adjuvant surgery in testicular cancer patients undergoing postchemotherapy retroperitoneal lymph node dissection. Ann Surg Oncol 2012; 19(07):2388-2393

16 Oldenburg J, Alfsen GC, Lien HH, Aass N, Waehre H, Fossa SD. Postchemotherapy retroperitoneal surgery remains necessary in patients with nonseminomatous testicular cancer and minimal residual tumor masses. J Clin Oncol 2003;21(17):3310-3317

17 Ozen H, Ekici S, Sozen S, Ergen A, Tekgül S, Kendi S. Resection of residual masses alone: an alternative in surgical therapy of metastatic testicular germ cell tumors after chemotherapy. Urology 2001;57(02):323-327

18 Vergouwe Y, Steyerberg EW, Foster RS, Habbema JD, Donohue JP. Validation of a prediction model and its predictors for the histology of residual masses in nonseminomatous testicular cancer. J Urol 2001;165(01):84-88, discussion 88

19 Leão R, Nayan M, Punjani N, et al. A New Model to Predict Benign Histology in Residual Retroperitoneal Masses After Chemotherapy in Nonseminoma. Eur Urol Focus 2018;4(06): 995-1001

20 Carver BS, Bianco FJ Jr, Shayegan B, et al. Predicting teratoma in the retroperitoneum in men undergoing post-chemotherapy retroperitoneal lymph node dissection. J Urol 2006;176(01): 100-103, discussion 103-104 